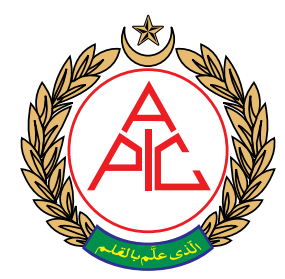

${ }^{1}$ Assistant Professor, Department of Anesthesia and Critical Care, King

Abdulaziz University Hospital, Jeddah (Saudi Arabia)

${ }^{2}$ Endocrinology Fellow, Department of Medicine, King Abdulaziz University Hospital, Jeddah (Saudi Arabia)

${ }^{3}$ Senior Registrar of Internal Medicine and Adult Critical Care, Department

of Anesthesia and Critical Care, King

Abdulaziz University Hospital, Jeddah

(Saudi Arabia)

Correspondence:

Dr Haifa Mesfer Algethamy,

Assistant Professor \& Consultant

Anesthesia \& Critical Care.

Department of Anesthesia \& Critical

Care, King Abdulaziz University

Hospital, Jeddah (Saudi Arabia).

E-mail: halgethamy2020@gmail.com

Received: 1 December 2019,

Reviewed: 11 December 2019, 2

January 2020,

Accepted: 10 January 2020

\section{A case of severe hypercalcemia with arterial and venous thrombosis}

\author{
Haifa Mesfer Algethamy ${ }^{1}$, Yasamen Abdulmannan Shikdar², \\ Tariq A. Alansari ${ }^{3}$
}

\begin{abstract}
We present a case of severe hypercalcemia with extensive venous and arterial thrombosis that led to the patient's demise in the setting of possible multiple endocrine neoplasia (MEN) type 2a. A 35-year-old female presented to the emergency with nausea and vomiting for one week. Physical examination revealed dry mucous membranes, a thyroid nodule on left side and epigastric tenderness. Initial investigations revealed evidence of renal impairment and hypercalcemia. Parathyroid hormone (PTH) level was very high. Ultrasound of the thyroid showed a solitary left thyroid nodule with mixed cystic and solid isoechoic echogenicity. The patient developed progressive dyspnea and hypoxemia, which mandated mechanical ventilation. Dialysis was initiated via the right femoral catheter and stopped due to extensive venous thrombosis of the right lower limb. Pulmonary emboli were excluded and pulmonary edema was confirmed by computed tomography. The patient was subsequently intubated for persistent respiratory distress. The same condition occurred in the right upper limb. Fine needle biopsy of the left thyroid nodule revealed medullary thyroid cancer. The consulting team preferred to manage her conservatively as she was rapidly-deteriorating. She developed progressive shock and multi-organ failure and expired.
\end{abstract}

Key words: Hypercalcemia; Hyperparathyroidism; Thrombosis; Limb ischemia; Medullary thyroid cancer; Multiple endocrine neoplasia

Citation: Algethamy HM, Shikdar YA, Alansari TA. A case of severe hypercalcemia with arterial and venous thrombosis. Anaesth pain intensive care 2020;24(1):111114. DOI: https://doi.org/10.35975/apic.v24i1.1235

\section{INTRODUCTION}

Hypercalcemia is a common medical condition. Patients usually present with mild symptoms like fatigue, abdominal pain, and constipation; but rarely, they can present as an endocrine emergency with convulsions, confusion or coma. ${ }^{1}$ It can be caused by a variety of disorders, like hyperthyroidism and hyperparathyroidism, both solid and hematologic malignancies, granulomatous diseases and renal diseases; or they can simply be due to exogenous calcium intake or drug induced. It has been reported that severely-elevated levels of ionized calcium can very rarely lead to vascular hypercoagulability and thrombosis. $^{2-7}$

Herein, we present a case of severe hypercalcemia with extensive venous and arterial thrombosis that led to the patient's demise in the setting of possible multiple endocrine neoplasia (MEN) type 2a.

\section{CASE REPORT}

A 35-year-old female patient presented to the emergency department with a history of nausea and vomiting for one week. Her past medical history only was significant for an abortion. Upon presentation, she was alert and oriented and her vital signs were significant for a postural drop in blood pressure and tachycardia. Physical examination was remarkable for dry mucous membranes, a left thyroid nodule and epigastric tenderness. Initial investigations revealed evidence of renal impairment and hypercalcemia. Creatinine was $228 \mu \mathrm{mol} / \mathrm{L}$, urea $7.9 \mathrm{mmol} / \mathrm{L}$, phosphate $1.93 \mathrm{mmol} / \mathrm{L}$, calcium $4.28 \mathrm{mmol} / \mathrm{L}$, and albumin $27 \mathrm{~g} / \mathrm{L}$. Other laboratory investigations showed a normal blood count, sodium 136, and potassium $3.3 \mathrm{mmol} / \mathrm{L}$, with normal serum amylase and lipase levels of $50 \mathrm{U} / \mathrm{L}$ and $204 \mathrm{U} / \mathrm{L}$ (Normal levels 0-137 and 12-70 U/L), respectively. Urine $\mathrm{Na}$ and creatinine were consistent with a renal cause of renal failure as fractional excretion of sodium $(\mathrm{FENa})>$ 
2\%). Parathyroid hormone (PTH) level was very high at $232 \mathrm{pmol} / \mathrm{L}$. Thyroid stimulating hormone (TSH) level was normal at $0.409 \mathrm{mU} / \mathrm{L}$ and a pregnancy test was negative. Urine analysis was positive for WBC 45/ hpf and RBC 6/hpf, but negative for nitrites.

The patient was admitted to the medical floor and was commenced on aggressive intravenous (IV) hydration and ciprofloxacin with a presumptive diagnosis of PTH-dependent hypercalcemia complicated by acute kidney injury and possible urinary tract infection. Ultrasound of the abdomen and pelvis was normal, apart from gallbladder sludge with mild distension. Both kidneys were normal in size and echotexture. Neither obstruction nor hydronephrosis was reported. Urine and blood cultures came back negative, so ciprofloxacin was discontinued. The patient received one dose of denosumab.

Ultrasound of the thyroid showed a solitary left thyroid nodule (Figure 1a) measuring $4.8 \times 2.7 \mathrm{~cm}$ with mixed cystic and solid isoechoic echogenicity, and a fine needle aspiration (FNA) biopsy of this nodule was planned. However, on the fourth admission day, the patient developed progressive dyspnea and needed to be maintained on supplemental oxygen via a nasal cannula. Chest radiographs (Figure 1b) demonstrated mild diffuse alveolar opacities. The patient was kept on IV fluids because of severe hypercalcemia, but received intermittent furosemide doses and a ventilation/perfusion (V/Q) scan was arranged, which was interpreted as a low probability of pulmonary embolism.

Nevertheless, the patient continued to deteriorate with progressive dyspnea and hypoxemia, along with severe tachycardia requiring intensive care unit (ICU) admission and mechanical ventilation support. IV fluids were held as the patient had evidence of pulmonary edema. Diuretics and broad-spectrum antibiotics were started. Calcitonin was started at 200 iu every $12 \mathrm{~h}$ BID and increased to 400 iu BID. Echocardiography was normal.

However, the serum calcium level remained severely elevated at $4.66 \mathrm{mmol} / \mathrm{L}$; thus, a decision to initiate dialysis via a right femoral catheter was made. A few hours following the end of dialysis, the patient's right lower limb was seen to show swelling and discoloration and the patient started complaining of severe pain. Bedside ultrasound confirmed right femoral vein deep venous thrombosis (DVT) (Figure 2a). The catheter was removed promptly, and a heparin infusion was initiated. The patient was subsequently intubated for persistent respiratory distress. Computed tomography (CT) pulmonary angiography (CTPA) was negative for pulmonary emboli, but revealed diffuse ground glass opacities suggestive of pulmonary edema (Figure 2b). Computed tomography of the right lower limb showed extensive venous thrombosis involving the bifurcation of the inferior vena cava (IVC), right common iliac vein, and right external iliac vein and its branches. Extensive soft tissue edema also was noted. The arterial system was patent.

To continue the dialysis sessions to overcome refractory hypercalcemia, a dialysis catheter was placed in right internal jugular, and the patient had her second dialysis session. A few hours after this second session, she was noted to have progressive cyanosis of the distal right upper limb and a weak radial pulse (Figure 2c). CT angiogram of the right upper limb confirmed total occlusion of the right proximal radial artery and right mid ulnar artery. The patient was still on heparin with a therapeutic partial thromboplastin time (PTT). A thrombophilia screen including antiphospholipid antibody syndrome (APAS) was requested. The vascular surgery service elected not to intervene, due to the critical condition of the patient and multiple vessels involvement.

Following a multidisciplinary team discussion involving the ICU, endocrinology, hematology, rheumatology and vascular services, a tentative diagnosis of catastrophic anti-phospholipid anti-body syndrome (APAS) was made, on a background of undiagnosed malignancy, like thyroid or parathyroid malignancy. Pulse steroid (inj methylprednisolone 1 g) daily for three days and plasma exchange sessions daily were initiated.

Both the right upper and lower limbs continued to get worse with extensive swelling and discoloration, despite the therapeutic PTT targets upon heparin infusion. The patient developed compartment syndrome in her right lower limb. Her total creatinine kinase $(\mathrm{CK})$ reached above $14000 \mathrm{u} / \mathrm{L}$. Orthopedic surgeons decided to manage conservatively, due to the patient's rapidly-deteriorating course.

During her ICU stay, her calcium level came down to normal ranges on dialysis. Thrombophilia screen and APAS antibodies were negative. Fine needle biopsy of the left thyroid nodule yielded malignant cells, which were interpreted as likely medullary thyroid cancer, but also potentially parathyroid malignancy. RET oncogene and calcitonin testing were postponed, due to the patient's critical condition.

She remained in the ICU intubated for a week afterwards and was shifted from dialysis to continuous renal replacement therapy. Her condition continued to deteriorate further, including progressive shock and multi-organ failure. Death later ensued.

\section{DISCUSSION}

Our case was a patient with medullary thyroid carcinoma (MTC) based on FNA, who presented with severe hypercalcemia that required sessions of dialysis, complicated by upper and lower limb venous/ arterial thrombosis. Unfortunately, she experienced a 
rapidly-deteriorating course with progressive shock and multi-organ failure, ultimately leading to death.

MTC is one of the neuroendocrine tumors that grows out of specialized thyroid cells called parafollicular or C cells, which synthesize a hormone called calcitonin. Calcitonin levels are used to help diagnose medullary thyroid cancer and as a marker for recurrence. Most MTCs are sporadic; but between 5 and $25 \%$ are familial, as part of multiple endocrine neoplasia type 2 (MEN2) syndrome. ${ }^{3-6}$ The diagnosis of MTC usually requires a combination of blood tests, imaging with ultrasound, and confirmation by histopathology of thyroid nodules by FNA biopsy.

In normal homeostasis, we need free ionized calcium for the initial steps of platelet plug formation and its use in multiple processes along the blood coagulation cascade. Calcium ions have known roles during clot formation and acceleration, combining with prothrombin to form thrombin, a proteolytic enzyme that, in turn, acts on fibrinogen to form fibrin monomers, the aggregation of which will form a final insoluble fibrin clot.

The link between hypercalcemia and thrombosis is still not completely understood and, due to the rarity of such cases, data are scarce. Possible mechanisms that have been proposed start with the coagulation cascade and its role in fibrin clot formation. ${ }^{7}$ However, calcium also can effect blood vessels via calcification and vasoconstriction, triggering vascular smooth muscle contraction due to increased intracellular free calcium levels. Furthermore, direct renal vasoconstriction can lead to dehydration and a further hypercoagulable state. ${ }^{8-9}$

A hypercoagulable state is common with malignancies, but how much this risk is altered if it is accompanied by severe hypercalcemia remains unknown. A review of published case reports about the correlation between severe hypercalcemia and thromboembolism showed that there is an increased risk of thrombosis. The search uncovered just five case reports, however, illustrating thrombosis in different sites with an underlying parathyroid adenoma and severe hypercalcemia, as well as a single case due to an underlying parathyroid carcinoma.

To the best of our knowledge, there is no report on the development of upper and lower limb thrombosis in a patient with hypercalcemia. Other cases reported in the literature were those five patients with an underlying parathyroid adenoma, all of whom had calcium levels above $3 \mathrm{mmol} / \mathrm{L}$, in addition to high PTH levels. Some of them, like our patient, had very acute hospital course which ended in patient demise. Two of the patients had a lower DVT, one of them catastrophic femoral DVT which led to muscular necrosis and subsequent multi-organ failure and death. ${ }^{10}$ Another patient presented with acute right-sided chest pain, in whom pulmonary CT angiography confirmed pulmonary emboli. One patient presented with left-sided hemiplegia and a right gaze palsy, while brain MRI revealed a cerebral infarction. ${ }^{2}$ The last patient presented with unilateral visual loss, which ultimately was attributed to optic neuropathy secondary to arterial ischemia. ${ }^{11}$ All these patients underwent surgical treatment of their hyperparathyroidism and, following surgery, had their PTH and calcium levels return to normal. A sixth report described a patient with bone pain, constipation and polyuria; on examination had a fixed neck mass. The patient was confirmed to have a parathyroid carcinoma. A pre-operative CT scan of the neck and chest revealed incidental filling defects in the right subclavian vein and in a branch of the left pulmonary artery indicating right subclavian vein thrombosis and a left pulmonary artery embolism, respectively. As with the others, this patient went for surgical resection of his tumor, which resulted in cure. $^{12}$

\section{CONCLUSIONS}

Severe hypercalcemia is one of the rare causes of arterial/venous thrombosis, though the mechanism behind hypercoagulability from hypercalcemia is still not well understood. We suggest checking calcium levels in patients with an unexplained hypercoagulable state. Prompt recognition and management of such cases is crucial.

\section{Competing Interests:}

The authors declare that they have no competing interests.

Authors' contributions:

All authors took part in the management, and wrote and approved the final manuscript. 


\section{REFERENCES}

1. Shane E, Irani D. Hypercalcemia: Pathogenesis, clinical manifestations, differential diagnosis, and management. In: Primer on the Metabolic Bone Diseases and Disorders of Mineral Metabolism, $6^{\text {th }}$ edition, Favus MJ (Ed), American Society for Bone and Mineral Research, Washington, DC 2006.

2. Koufakis $\mathrm{T}$, Antonopoulou $\mathrm{V}$, Grammatiki M, Karras SN, Ajjan R, Zebekakis P, et al. The Relationship between Primary Hyperparathyroidism and Thrombotic Events: Report of Three Cases and a Review of Potential Mechanisms. Int J Hematol Oncol Stem Cell Res. 2018 Jul 1;12(3):175180. [PubMed] [Free Full Text]

3. Saad MF, Ordonez NG, Rashid RK, Guido JJ, Hill CS Jr, Hickey $\mathrm{RC}$, et al. Medullary carcinoma of the thyroid. A study of the clinical features and prognostic factors in 161 patients. Medicine (Baltimore). 1984;63(6):319. [PubMed]

4. Dottorini ME, Assi A, Sironi M, Sangalli G, Spreafico G, Colombo L. Multivariate analysis of patients with medullary thyroid carcinoma. Prognostic significance and impact on treatment of clinical and pathologic variables. Cancer. 1996;77(8):1556. [PubMed]

5. Gagel RF, Hoff A0, Cote GJ. Medullary thyroid carcinoma. In: Werner \& Ingbar's The Thyroid, 9th, Braverman LE, Utiger RD (Eds), Lippincott Williams \& Wilkins, Philadelphia 2005. p.967.

6. Kebebew E, Ituarte $\mathrm{PH}$, Siperstein $\mathrm{AE}$, Duh QY, Clark OH. Medullary thyroid carcinoma: clinical characteristics, treatment, prognostic factors, and a comparison of staging systems. Cancer. 2000 Mar;88(5):113948. [PubMed]

7. Erem C, Kocak M, Nuhoglu, I, Yilmaz M, Ucuncu 0 . Increased plasminogen activator inhibitor-1, decreased tissue factor pathway inhibitor, and unchanged thrombin-activatable fibrinolysis inhibitor levels in patients with primary hyperparathyroidism. Eur J Endocrinol. 2009;160(5):8638. [PubMed] DOI: 10.1530/EJE-09$\underline{0069}$.

8. Walker GL, Williamson PM, Ravich
RB, Roche J. Hypercalcaemia associated with cerebral vasospasm causing infarction. J Neurol Neurosurg Psychiatry. 1980;43(5):464-467. [PubMed] [Free Full Text]

9. Yarnell PR, Caplan LR. Basilar artery narrowing and hyperparathyroidism: illustrative case. Stroke. 1986;17(5):1022-4. [PubMed]

10. Lin Chen, Zhongheng Zhang. Catastrophic femoral vein thrombosis in a patient with hypercalcemia due to parathyroid adenoma: a case report. J Emerg Crit Care Med 2017;1:2. [Free Full Text] DOI: 10.21037/ jeccm.2017.02.02

11. Chappel D, Farrington K. Primary hyperparathyroidism presenting as unilateral visual loss. Postgrad Med J. 1991 May;67(787):469-70[PubMed] [Free Full Text]

12. Manosroi W, Wannasai $\mathrm{K}$, Phimphilai M. Pulmonary Embolism and Subclavian Vein Thrombosis in a Patient with Parathyroid Carcinoma: Case Report and Review of Literature. J Med Assoc Thai.2015;98(9):925-33 [PubMed]

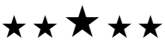

